\title{
Le dévouement à la nation. Les combats d'idées entre 1911 et 1914
}

Hingabe an die Nation. Die Ideenkämpfe 1911-1914

Devotion to the nation. The "battle " of ideas between 1911 and 1914

\section{Gangolf Hübinger}

Traducteur : Julien Thys

\section{QpenEdition}

\section{Journals}

Édition électronique

URL : http://journals.openedition.org/ceg/1913

DOI : $10.4000 /$ ceg. 1913

ISSN : 2605-8359

\section{Éditeur}

Presses Universitaires de Provence

Édition imprimée

Date de publication : 15 juin 2014

Pagination : 29-38

ISSN : 0751-4239

Référence électronique

Gangolf Hübinger, «Le dévouement à la nation. Les combats d'idées entre 1911 et 1914 », Cahiers d'Études Germaniques [En ligne], 66 | 2014, mis en ligne le 17 décembre 2017, consulté le 21 novembre 2020. URL : http://journals.openedition.org/ceg/1913; DOI : https://doi.org/10.4000/ceg.1913 


\title{
Le dévouement à la nation. Les combats d'idées entre 1911 et 1914
}

\author{
Gangolf HÜBINGER \\ Europa-Universität Viadrina, Frankfurt/Oder
}

Pendant les années qui précédèrent la Première Guerre mondiale, les sociétés européennes se trouvèrent engagées, à l'échelle du globe, dans des «zones de contact» transnationales toujours plus larges ${ }^{1}$. Ce processus renforça la rivalité des États-nations modernes et promut des idées et des représentations du monde qui, dans un registre martial, interrogeaient la capacité de la "force de la nation » à s'imposer dans la compétition mondiale ${ }^{2}$. Des combats d'idées agonistiques préfigurèrent le combat militaire.

La formule du "dévouement à la nation » (Hingabe an die Nation), caractéristique de l'époque et qui sert de point de départ à cet article, se trouve chez le théologien, philosophe de la culture et journaliste Ernst Troeltsch. C'est Troeltsch qui a forgé la formule percutante de "guerre des cultures ». Il prononça son discours le plus significatif sur ce thème en mars 1916 devant la Deutsche Gesellschaft 1914 (« Société allemande 1914 »), et le fit ensuite publier dans la Neue Rundschau en mai 1916, dans une version très augmentée, sous le titre programmatique : «Les idées de 1914 ». La Neue Rundschau comptait parmi les revues littéraires de premier plan et était publiée par le S. Fischer-Verlag, une maison d'édition de renom. Troeltsch y dressait consciencieusement l'inventaire des «idées de 1914 » que les intellectuels allemands avaient, après le $1^{\text {er }}$ août 1914 , mobilisées contre les idées occidentales de 1789 et énonçait en premier lieu la « renaissance de la

\footnotetext{
${ }^{1}$ Emily S. RoSENBERG, «Transnationale Strömungen in einer Welt, die zusammenrückt 》 (Courants transnationaux dans un monde interdépendant), in ID., et al. (dir.), Geschichte der Welt 1870-1945. Weltmärkte und Weltkriege (Histoire mondiale 1870-1945. Marchés mondiaux et guerres mondiales), München, Beck, 2012, p. 815-998, p. 821.

Dieter LANGEWIESCHE, " Das Jahrhundert Europas. Eine Annäherung in globalgeschichtlicher Perspektive » (Le siècle de l'Europe. Une approche du point de vue de l’Histoire globale), Historische Zeitschrift, n 296, 2013, p. 29-48, p. 32.
} 
subjectivité, qui avait dégénéré en culte de soi, dans le libre dévouement à l'esprit national ${ }^{3} »$.

Il convoque ici le tout jeune esprit national de 1813 dans sa dimension historico-politique. Il fallait, en 1914, recréer et réactualiser la mobilisation de la nation allemande contre les ambitions impériales de Napoléon, car, d'après Troeltsch", qui se conformait ici à l'opinion majoritaire en Europe, «la situation d'aujourd'hui n'est en rien différente ». À partir du $1^{\text {er }}$ août, les intellectuels européens ne se livrèrent plus seulement à une lecture dualiste du temps présent, mais également de l'Histoire européenne tout entière en identifiant deux pôles clairement opposés : amis et ennemis. C'est l'appel, souvent cité, d'Henri Bergson à la « lutte de la civilisation contre la barbarie », adressé à l'Académie des sciences morales et politiques ${ }^{5}$. Pour Werner Sombart, sociologue influent, la guerre opposait des héros allemands à des marchands anglais ${ }^{6}$. Les innombrables appels à la guerre de la race germanique contre la race slave exercèrent également un fort pouvoir d'identification. Les représentations manichéennes engendrées par la « guerre des cultures » et apparues après le $1^{\mathrm{er}}$ août 1914 ne sont cependant pas ici notre sujet. Troeltsch, quant à lui, considérait déjà cette époque comme bien trop complexe, compte tenu de la « politique internationale planétaire ${ }^{7}$ (ainsi désignait-il le processus de globalisation qui s'accomplissait au seuil du $\mathrm{XX}^{\mathrm{e}}$ siècle), pour pouvoir être lue en fonction de schémas aussi simples. Son propos est autre. Troeltsch mobilise ses lecteurs, convaincu que, si les Français et les Anglais disposent déjà des modèles agonistiques d'une guerre

\footnotetext{
${ }^{3}$ Voici l'intégralité du passage, qui se distingue par son emphase religieuse : «Éducation populaire basée sur les méthodes de Pestalozzi, autarcie de l'État en tant qu'État commercial fermé, renaissance de la subjectivité, qui avait dégénéré en culte de soi, dans le libre dévouement à l'esprit national, accomplissement de l'esprit national à partir du mouvement propre au devenir divin de l'univers, reconnaissance réciproque des esprits nationaux comme miroirs de la divinité. En résumé : la prise de conscience de l'essence à l'origine libre et productrice de la germanité face à celle artificielle, dérivée, décorative et rationaliste de la romanité - telles étaient autrefois les idées nouvelles. », Ernst TrOELTSCH, « Die Ideen von 1914 »(Les idées de 1914), Die neue Rundschau, 27, 1916, p. 605-624, édition abrégée in Deutscher Geist und Westeuropa (L'esprit allemand et l'Europe de l'ouest), Tübingen, Mohr, 1925, p. 31-58, cité ici d'après l'édition intégrale du texte paru dans la Neue Rundschau, cit. p. 608. La désignation de la guerre comme « guerre des cultures » se trouve ibid., p. 607.

${ }^{4}$ Ibid., p. 608

5 Cité dans Michel WINOCK, Das Jahrhundert der Intellektuellen (Le siècle des intellectuels), Constance, UVK-Verlagsgesellschaft, 2003, p. 173.

${ }^{6}$ Werner SOMBART, Händler und Helden. Patriotische Besinnungen (Marchands et héros. Réflexions patriotiques), München/ Leipzig, Duncker \& Humblot, 1915 ; sur l'intégration européenne, cf. Hans JOAS, «Die Sozialwissenschaften und der Erste Weltkrieg. Eine vergleichende Analyse » (Les sciences sociales et la Première Guerre mondiale. Une analyse comparée), in Wolfgang J. MOMMSEN, Kultur und Krieg. Die Rolle der Intellektuellen, Künstler und Schriftsteller im Ersten Weltkrieg (Guerre et culture. Le rôle des intellectuels, artistes et écrivains pendant la Première Guerre mondiale), München, Oldenbourg, 1996, p. 17-29.

${ }^{7}$ TROELTSCH, Die Ideen von 1914, p. 609.
} 
culturelle, les Allemands, dans leur détresse existentielle, doivent encore les faire surgir du sein du « creuset de la grande catastrophe ${ }^{8}$ ».

Troeltsch, en comparant ainsi les idées politiques allemandes à celles des ennemis, attire le regard sur la phase d'avant-guerre. Jusqu'à quel point les élites culturelles européennes pensent-elles sur le mode de la guerre culturelle et jusqu'à quel point les intellectuels qui auscultent leur époque rivalisent-ils entre eux avant d'avoir vécu le $1^{\text {er }}$ août 1914 ? Comment les experts de l'observation et de la description des sociétés contemporaines, les historiens du temps présent, sociologues, philosophes et journalistes politiques, se représentent-ils la valeur et la place de leur nation à l'intérieur du système de la « politique internationale planétaire »? En d'autres termes, quelles idées et quels combats d'idées conduisent la partie de l'opinion publique qui réfléchit sur son époque à se déclarer prête à la guerre ou à s'y résigner avec fatalité, sans savoir que l'un de ces conflits régionaux et l'une de ces nombreuses crises diplomatiques ont effectivement enclenché le processus inexorable de la mobilisation?

L'histoire des conflits européens identifie un moment particulier où l'antagonisme des idées politiques devint si fort que la perspective de la guerre, particulièrement en France et en Allemagne puis, par ricochet, en Angleterre, Autriche-Hongrie et Russie, s'imposa définitivement dans les esprits. Entre juillet et octobre 1911, la deuxième crise marocaine, conséquence de la diplomatie allemande de la canonnière durant le « coup d'Agadir ", conduisit l'Europe plus près de la guerre qu'aucune des crises précédentes. Les journaux allemands, depuis les feuilles à grand tirage jusqu'aux revues littéraires trimestrielles, notamment Zukunft («L'Avenir ») de Maximilian Harden, déclenchèrent alors une campagne nationaliste qui, sur un ton agressif, alla jusqu'à envisager ouvertement le déclenchement de la guerre.

C'est à la «Nation » qu'on en appelait de manière toujours plus incantatoire, comme au dernier rempart contre les crises extérieures ou intérieures. Lorsqu'un Européen se référait après 1911 à la « Nation » en tant que communauté politique, il comprenait celle-ci comme une communauté guerrière qui exigeait un dévouement allant jusqu'au sacrifice ultime. Dans l'espace public de la communication, il argumentait en recourant à des codes binaires : nous et les autres, amis et ennemis, sécurité et menace, voire progrès et déclin.

Les recherches actuelles confirment le principe de la thèse plus ancienne de Wolfgang J. Mommsen, selon laquelle c'est à ce moment-là que l'idée de l'« inéluctabilité » d'une grande guerre européenne, d'une guerre mondiale s'ancra définitivement dans les esprits et détermina par la suite les politiques des nations. Une idée qui fut moins imposée par une hiérarchie militaire aveugle ou des «cliques réactionnaires » que par l'opinion publique et ses têtes pensantes relayées par les médias modernes. Les acteurs politiques de la

${ }^{8}$ Ibid., p. 608. 
crise de juillet se seraient ainsi soumis à la fatalité de la guerre et leur participation intransigeante à l'aggravation de la crise aurait été «la conséquence de deux décennies d'agitation nationaliste que la politique officielle n'a jamais été en mesure de contenir ${ }^{9} »$. Le dernier livre paru sur ce thème, Sleepwalkers, de Christopher Clark, fait certes état de voix plus mesurées, notamment en Angleterre ${ }^{10}$. Mais le climat politique général était marqué par "un antagonisme extrême et le sentiment d'une impasse ", qu'illustrait une "représentation toujours plus négative des "forces ennemies" ». La pression des journaux rendit la « marge de manœuvre des acteurs politiques [...] toujours plus étroite ${ }^{11} »$.

Je m'intéresse cependant moins ici à l'opinion collective et anonyme véhiculée par les médias qu'aux élites qui posèrent un diagnostic sur leur époque. Jouissant du statut exposé de porte-parole, ces dernières étaient considérées comme les " guides spirituels de la nation ${ }^{12}$ », qu'elles donnent des représentations nettement orientées de soi et des autres, ou qu'elles imposent en les rationalisant systématiquement des systèmes de domination nationale. Or, au plus tard à partir de 1911, cette Europe des élites entra dans une période de violente agitation.

En France, un nouveau courant réunissant des écrivains comme Charles Péguy, Henri Massis et Alfred de Tarde gagna en influence aux dépens de l'ancienne opposition des «deux Frances », celle des Dreyfusards et celle de l'Action française. Elle fit en sorte d'entraîner la jeunesse universitaire dans les « rouages du nationalisme » et de freiner la politique de conciliation d'un Jean Jaurès ${ }^{13}$. Dans l'Empire allemand aussi, une troisième force s'affirmait, face aux bellicistes, rassemblés traditionnellement au sein de la Ligue pangermaniste, et aux opposants à la guerre, partisans de la social-démocratie. Ces voix en faveur d'un libéralisme impérial se firent plus fortes et, phénomène propre à la culture allemande, ce ne furent pas, comme en France, les écrivains qui imposèrent le ton nationaliste, mais les professeurs politisés.

${ }^{9}$ Wolfgang J. Mommsen, « Der Topos vom unvermeidlichen Krieg: Außenpolitik und öffentliche Meinung im Deutschen Reich im letzten Jahrzehnt vor 1914 » (Le topos de la guerre inéluctable : politique étrangère et opinion publique dans l'Empire allemand pendant la dernière décennie avant 1914), in id., Der autoritäre Nationalstaat. Verfassung, Gesellschaft und Kultur im deutschen Kaiserreich (L'État-nation autoritaire. Constitution, société et culture dans l'Empire allemand), Frankfurt am Main, Fischer Taschenbuch, 1990, p. 380-406, p. 405 sq.

Christopher CLARK, The Sleepwalkers. How Europe went to War in 1914 (Les somnambules. Comment l'Europe entra en guerre en 1914), London, Penguin Books, 2013, surtout p. 236.

${ }^{11}$ Clark s'appuie également sur les résultats de l'analyse détaillée de la presse menée par Bernhard ROSENBERgER, Zeitungen als Kriegstreiber? Die Rolle der Presse im Vorfeld des Ersten Weltkrieges (Les journaux comme va-t-en-guerre ? Le rôle de la presse avant la Première Guerre mondiale), Köln, Böhlau, 1998.

${ }^{12}$ Cf. Kurt Flasch, Die geistige Mobilmachung. Die deutschen Intellektuellen und der Erste Weltkrieg. Ein Versuch (La mobilisation savante. Les intellectuels allemands et la Première Guerre mondiale. Essai), Berlin, Alexander Fest, 2000, p. 7.

13 Joseph JURT, Frankreichs engagierte Intellektuelle. Von Zola bis Bourdieu (Les intellectuels engagés en France. De Zola à Bourdieu), Göttingen, Wallstein Verlag, 2012, p. 59. 
C'est à Fribourg, paisible ville universitaire du sud-ouest libéral de l'Allemagne située près de la frontière française, que l'historien Friedrich Meinecke prononça, en juin 1913, un discours officiel intitulé Deutsche Jahrhundertfeier und Kaiserfeier («Fête allemande du centenaire et fête de l'empereur ») à l'occasion du double jubilé Hundert Jahre Befreiungskriege («Centenaire des guerres d'indépendance ») et Fünfundzwanzig Jahre Regentschaft Wilhelms $I^{14}$ ( ( Les 25 ans de la régence de Guillaume II »). Meinecke, en rédigeant en 1907 Weltbürgertum und Nationalstaat (« Cosmopolitisme et État-nation »), s'était hissé au premier rang des historiens allemands de l'ère moderne. Dans sa préface à la deuxième édition de 1911, il décrivait ainsi le but de son ouvrage : que la recherche historique allemande, « de son propre chef et de concert avec les grandes forces de l'État et de la vie culturelle ", " ose davantage s'aventurer dans les domaines philosophiques et politiques, car c'est ainsi seulement qu'elle atteindra à sa véritable essence, qui consiste à être à la fois universelle et nationale ${ }^{15} \%$. Quant à s'aventurer audacieusement en politique, le discours fribourgeois du jubilé de 1913 contenait en effet à la fois un sous-texte agonistique et un message explicitement agonistique.

Au-delà de la question centrale «Qu'est-ce que l'essence d'une nation ? ", le texte contenait en filigrane une attaque contre la France : l'« orgueil rationaliste de la Révolution française », associé à l'expansion de la puissance impérialiste sous Napoléon, avait fait de la France une puissance étatique nationale et européenne menaçante. Les Allemands, quant à eux, privilégiaient la " belle âme romantique ${ }^{i 6}$ ", une conception du monde à michemin entre la mise en avant d'un " moi créateur» et l'idéalisation emphatique de l'Humanité. Entre les deux nations existait un dangereux vide politique. Dans leur combat contre Napoléon, les Allemands avaient enfin pris conscience que le " souci de la préservation de leur existence » exigeait l'unité politique d'un État-nation capable de se défendre "grâce à l'épanouissement de toutes les forces de la volonté et de l'esprit qui composent une nation, par la préservation sans concession de toutes ses conquêtes et, si besoin est, au travers d'une lutte sanglante et acharnée ${ }^{17} \gg$. C'est avec une certaine habileté rhétorique que Meinecke associa le lieu mémoriel de 1813 à la situation conflictuelle de 1913, lorsqu'il loua l'Empereur comme faisant désormais figure de garant d'une nation culturelle économiquement prospère et militairement forte, et ce, dans un temps où, tout comme en 1813, les nations « entraient dans une zone de danger dont l'issue se trouvait plongée dans les ténèbres ». Le diagnostic de Meinecke sur son

\footnotetext{
14 Friedrich MeINECKE, « Deutsche Jahrhundertfeier und Kaiserfeier. Freiburger Universitätsrede 14. Juni 1913 » (Fête allemande du centenaire et Fête de l'Empereur, discours tenu à l'université de Fribourg le 14 juin 1913), Logos 4, 1913, p. 161-175.

${ }^{15}$ Friedrich MeINECKE, Weltbürgertum und Nationalstaat (Cosmopolitisme et État-nation), éd. par Hans Herzfeld, München, Oldenbourg, 1962, p. 2 sq.

${ }^{16}$ Ibid., p. 162, 166.

${ }^{17}$ Ibid., p. 162.
} 
époque se termine par le message explicite suivant : «Les véritables batailles de notre époque se trouvent, non pas derrière nous, mais devant nous $»^{18}$. Il convient de rappeler ici que Friedrich Meinecke entretenait avec Ernst Troeltsch, qu'il cite au début, un échange intellectuel intense. Ce potentiel agonistique que Troeltsch, dans ses « idées de 1914 », voyait seulement croître après le $1^{\mathrm{er}}$ août, Meinecke le réalisait déjà dans ses "idées de 1913 » en proposant un modèle d'interprétation élaboré apte à conduire l'action politique. En aucun cas il ne s'agissait d'un simple discours savant de circonstance.

Depuis un certain temps déjà, ces idées du dévouement guerrier à la Nation, cautionnées par l'autorité des professeurs, circulaient dans les milieux cultivés. À ce titre, la controverse née à la suite de la deuxième crise marocaine de novembre 1911, qui opposa l'université de Fribourg et le journal de la bourgeoisie de gauche, la Frankfurter Zeitung, apparaît comme exemplaire. Le vice-recteur de Fribourg avait profité de l'inauguration d'un nouveau bâtiment de l'université pour se livrer à des déclamations aux accents nationaux. La Frankfurter Zeitung écrivit dans son compte rendu: " Il mit alors en garde contre les "benêts", qui, avec leur "rengaine sur la paix", voulaient priver le peuple de ses défenses ». Ce journal de réputation internationale jugeait de tels propos malvenus et irresponsables, tout particulièrement lorsqu'ils étaient tenus devant la jeunesse universitaire. Or, des universitaires renommés protestèrent à leur tour dans un manifeste contre ce compte rendu critique. Parmi eux se trouvaient Georg von Below, Heinrich Rickert et Friedrich Meinecke ${ }^{19}$. Le philosophe Heinrich Rickert expliqua, dans une lettre à son ami Max Weber, qui vivait à Heidelberg, ce qui irritait tant les professeurs : «Ce qui m'a rendu ce journal si antipathique, c'est cette sorte de chauvinisme inversé qui dégénère à l'occasion en véritable police de la pensée, au point de pouvoir rivaliser avec les chauvinistes eux-mêmes ». Ainsi juge le premier des philosophes universitaires allemands. Et plus loin :

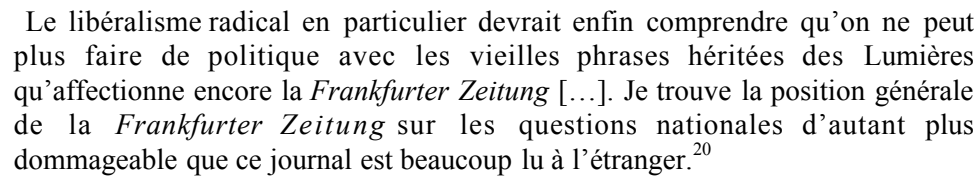

Les controverses autour de la montée en puissance de l'Empire allemand parmi les grandes puissances européennes dominaient le débat intellectuel et aucun autre thème n'était plus en mesure d'échauffer les esprits que celui de la « Nation ». Même un cercle de savants qui, dans ses statuts, s'était engagé

18 Ibid., p. 175.

19 Les faits sont relatés dans l'édition des Euvres complètes de Max Weber. Lettres 19111912, éd. par M. Rainer LEPSIUS et Wolfgang J. MOMMSEN, en collaboration avec Birgit RUDHARD et Manfred SCHÖN, Tübingen, Mohr, 1998 (MWG II/7), p. 337 sq.

20 Lettre d'Heinrich Rickert à Max Weber, non datée, UB Heidelberg, Heid.ms 2740 suppl. 93, 1.2. 
à l'objectivité la plus stricte ne fut pas épargné. En 1912, la deuxième « Journée de la sociologie allemande 1912 », qui se tenait à Berlin, plaça le thème de la « Nation » au centre de ses discussions. Un éclat s'ensuivit rapidement. Lorsque le philosophe Paul Barth prolongea ses réflexions dans le domaine de l'Histoire universelle sur le «Peuple» en tant qu' « organisme spirituel» par la question suggestive «Un État international ne serait-il pas plus bénéfique pour le progrès [...] qu'un État national ? », Max Weber provoqua l'interruption de l'intervention ${ }^{21}$. Weber, lui-même défenseur passionné de la consolidation de la puissance nationale, mettait en avant, sur un plan théorique, la nécessité d'expliquer " sans préjugé aucun » le " sentiment national » moderne. Il en proposait la définition suivante, qui suscita tout autant l'appui partiel que l'opposition d'interlocuteurs aussi illustres qu'Eduard Bernstein, Ludo Moritz Hartmann ou encore Robert Michels :

Si l'on considère comme pertinent d'identifier le sentiment national comme quelque chose d'homogène et de proprement spécifique, on ne peut le faire qu'en référence à un penchant pour son propre État, tout en étant conscient que s'y trouveront réunis des sentiments communautaires fortement hétérogènes quant à leur source et à leur nature. ${ }^{22}$

Une sociologie du nationalisme fondée sur une séparation entre science et politique n'était cependant plus en mesure de s'imposer. Les experts de la question sociale, les diagnosticiens de leur époque, cherchaient à les réunir et à « s'aventurer plus audacieusement" en politique, à la façon d'un Friedrich Meinecke. Défense des valeurs et professions de foi étaient les signes des temps nouveaux.

On trouve au sein du large spectre des élites libérales deux points de vue éminents qui illustrent cette situation, chacun lié à une revue influente. Il s'agit d'abord de la voix discrète, et par là sous-estimée, de l'historien des armées et éditeur des Annales prussiennes Hans Delbrück. Ce dernier prépare ses lecteurs à l'idée que la domination mondiale passera par des États-nations progressistes et qu' « il est impératif que l'opinion publique allemande comprenne » pourquoi l'Empire doit faire preuve d'une ambition plus forte « lors de la répartition coloniale du monde »:

Secteurs commerciaux, plantations, investissements de capitaux, exploitation de trésors naturels cachés. Il faut qu'un grand nombre d'Allemands puisse trouver dans les colonies l'occasion d'acquérir des biens ; ainsi, leur prospérité contribuera à la richesse de la nation dans son ensemble et profitera

\footnotetext{
21 Paul BARTH, « Die Nationalität in ihrer soziologischen Bedeutung » (La signification sociologique de la nationalité), in Verhandlungen des Zweiten Deutschen Soziologentages vom 20.-22. Oktober in Berlin (Discussions de la deuxième Journée de la sociologie allemande des 20 -22. octobre à Berlin), Tübingen, Mohr, 1913, p. 21-48, citation : dernière phrase p. 48. Le compte rendu indique : « ici, l'intervention a été interrompue. »

22 Max WEBER, «Diskussionsbeitrag im Anschluss an den abgebrochenen Vortrag von Paul Barth » (Intervention à l'issue du discours interrompu de Paul Barth), ibid., p. 52.
} 
indirectement aux masses au travers des profits économiques et des emplois qui en découleront. ${ }^{23}$

Une manière de voir caractéristique de l'impérialisme économique, mais qui n'est pas, pour l'auteur, décisive. Se référant à son collègue britannique, l'historien des cultures William Edward Hartpole Lecky qui vantait les mérites des colonies anglaises en tant que lieux d'apprentissage propres à forger le «British caracter », Delbrück conclut : « Ce qui est décisif, c'est que ce ne soit pas l'intérêt économique, mais l'idée nationale qui guide la politique coloniale». En effet, "le commerce et l'industrie ne sont que le moyen d'étendre l'expansion et la puissance du peuple allemand ${ }^{24} »$.

Delbrück évoquait avec enthousiasme le livre paru en 1912 du journaliste et écrivain-voyageur Paul Rohrbach : Der deutsche Gedanke in der Welt (La pensée allemande dans le monde). Delbrück souhaitait instamment que cette étude, qui professe que la nation, au travers de «l'idéal moral représenté par la culture allemande [...], doit apporter sa contribution au progrès de l'Humanité en marquant le monde du sceau de son idée nationale ${ }^{25} »$, connaisse « ce qu'on appelle une diffusion de masse ${ }^{26} »$. Ce souhait s'était déjà réalisé depuis longtemps. Le théologien et universitaire Rohrbach, dont la thèse percutante énonçait que le combat "pour l'avenir d'un peuple allemand au destin mondial » restait encore à mener ${ }^{27}$, était le plus retentissant et le plus lu des auteurs qui, au sein de la bourgeoisie allemande, défendaient l'idée d'une expansion de la mission culturelle de l'Allemagne. Il était présent dans toutes les revues spécialisées. Dans son diagnostic du temps présent à la dimension d'une encyclopédie «L'année 1913. Panorama de l'évolution culturelle », Rohrbach rendit ainsi accessible à un large lectorat les revendications d'une "expansion de nos domaines d'intérêt national», tout en avertissant que «nous [devions] être prêts, s'il n'y [avait] pas d'autre alternative, à nous battre pour cela ${ }^{28} \gg$.

Ainsi, le principe de la pensée impérialiste, selon lequel seules l'expansion armée et la pression exercée sur les rivaux de la scène politique mondiale permettent d'assurer l'existence d'une nation culturelle propre, se trouvait, de par la diffusion littéraire extraordinaire que lui conféraient des hommes

\footnotetext{
${ }^{23}$ Hans DELBRÜCK, « Über die Ziele unserer Kolonialpolitik » (Sur les buts de notre politique coloniale), Preußische Jahrbücher (Annales prussiennes), 147, 1912, p. 503-513, citations p. 504 sq.

${ }^{24}$ Ibid., p. 504.

25 Paul RohrBach, Der Deutsche Gedanke in der Welt (La pensée allemande dans le monde), Königstein im Taunus / Leipzig, Langewiesche, 1912, p. 6.

${ }^{26}$ Preußische Jahrbücher (Annales prussiennes), 148, 1912, p. 330-333, ici p. 330.

27 Cité ici à partir de l'ouvrage de Wolfgang J. MOMMSEN, Bürgerstolz und Weltmachtstreben. Deutschland unter Wilhelm II. 1890-1918 (Fierté citoyenne et désir de puissance mondiale. L'Allemagne sous Guillaume II), Berlin, Propyläen, 1995, p. 524.

28 Paul Rohrbach, «Welt- und Kolonialpolitik » (Politique mondiale et coloniale), in David SARASON (dir.), Das Jahr 1913. Ein Gesamtbild der Kulturentwicklung (L'année 1913. Une vue d'ensemble de l'évolution culturelle), Leipzig/ Berlin, B. G. Teubner, 1913, p. $45-53$, p. 52 .
} 
comme Paul Rohrbach, mis à portée de l'opinion publique allemande. Les années entre 1911 et 1914 furent dominées par ce principe, qui tirait sa force de l'étroite imbrication d'un ordre intérieur et extérieur découlant de la démocratisation croissante : «Promesses de participation à la vie de la nation et velléités d'agression ont toujours fait partie des fondements de la pensée nationale ${ }^{29} »$.

C'est justement dans cette optique que Paul Rohrbach et Ernst Jäckh, le promoteur des relations germano-turques, créèrent en avril 1914 l'hebdomadaire Das Größere Deutschland (L'Allemagne qui s'agrandit). Le premier cahier annonçait la collaboration régulière des plus célèbres professeurs et diagnosticiens de leur époque, depuis Rudolf Eucken jusqu'à Gustav von Schmoller en passant par Friedrich Meinecke ${ }^{30}$. On constate là encore que, si en France, c'étaient les écrivains qui donnaient la mesure de la mobilisation intellectuelle, ce rôle était tenu en Allemagne par les professeurs.

Quelle part joue l'Angleterre, alors première puissance mondiale, dans ce discours? La recherche souligne les fortes variations de l'opinion publique et une tendance marquée à détourner vers l'extérieur les menaces de guerre civile, comme lors du conflit sur la Home Rule irlandaise, au moyen d'appels militants à l'unité et à la force de la Nation. Sur ce front, la presse populaire, depuis le début du siècle, alimentait volontiers à coups de rhétorique guerrière les «newspaper wars", tout en ravivant parfois l'espoir d'une politique commune européenne tournée vers la paix. Le pouvoir de la presse modifia en tout cas la nature du combat d'idées public. À l'époque de Bismarck et de Salisbury déjà, l'opposition entre les intérêts nationaux allemands et britanniques était apparue clairement. La pression exercée par les journaux avant la Première Guerre mondiale changea donc moins les intérêts du moment que la « définition de ces intérêts sur la base des représentations dominantes de soi et du monde ${ }^{31} »$

Dans cette reformulation des intérêts politiques, les idées de nature agonistique, attisées par les intellectuels diagnosticiens de leur époque, revêtent une importance déterminante. Les public moralists anglais, de leur côté, observaient encore avec une certaine retenue cette guerre des esprits qui s'intensifiait sur le continent. C'est l'impression laissée par le $4^{\mathrm{e}}$ congrès international d'Histoire qui se tint en avril 1913 à Londres. Cependant, même à l'intérieur de cette "zone de contact" du savoir, régnait le sentiment latent d'une guerre inéluctable, et ce fut un intervenant allemand qui s'en fit l'écho.

\footnotetext{
${ }^{29}$ Dieter LangewIESChe, Reich, Nation, Föderation. Deutschland und Europa (Empire, Nation, Fédération. L'Allemagne et l'Europe), München, Beck, 2008, p. 55.

${ }^{30}$ Das Größere Deutschland, 1, 1914, p. 1.

${ }^{31}$ Puissamment illustré par Dominik GEPPERT, Pressekriege. Öffentlichkeit und Diplomatie in den deutsch-britischen Beziehungen, 1896-1912 (Guerres de la presse. Opinion publique et diplomatie dans les relations germano-britanniques), München, Oldenbourg, 2007, citation p. 436 ; cf. aussi J. Lee THOMPSON, Northcliffe. Press Baron in Politics, 1865-1922 (Northcliffe. Un baron de la presse en politique), London, John Murray, 2000, p. 201-221.
} 
La Frankfurter Zeitung rapporta que l'historien de la culture Karl Lamprecht avait lancé, pendant son discours sur les courants de pensée actuels de l'Empire, l'avertissement suivant : « Si, ce qu'il ne souhaitait pas, la guerre devait éclater, il faudrait s'attendre à trouver dans le peuple allemand un adversaire déterminés ${ }^{32} »$.

L'intervention de Karl Lamprecht, tout comme celles de Friedrich Meinecke, Hans Delbrück ou Paul Rohrbach, mais cette fois à l'étranger et devant un public international, montre la façon dont la "politique internationale planétaire » poussait les élites qui se penchaient sur leur époque à avancer des conceptions toujours plus bellicistes d'un ordre national. Avant le $1^{\text {er }}$ août 1914, elles s'efforçaient encore de prendre en considération les ramifications internationales d'une politique globalisée. Les modèles de pensée opposant les cultures nationales étaient cependant déjà si bien ancrés qu'ils purent, dès après le $1^{\text {er }}$ août, être exploités grâce à des images simplifiées du type : « démocratie occidentale » contre "idée allemande de liberté ». Ernst Troeltsch résuma tous ces arguments, d'une façon à la fois réfléchie et mobilisatrice, lorsque, au cours de la deuxième année de la guerre, opposant à sa manière les idées de 1914 aux idées de 1789, il en appela au devoir d'un « don religieux de sa personne ${ }^{33} »$.

(Traduction Julien Thys)

\footnotetext{
${ }^{32}$ Frankfurter Zeitung du 11. Avril 1913, Abendblatt, cit. d'après Karl Dietrich ERDMANN, Die Ökumene der Historiker. Geschichte der Internationalen Historikerkongresse und des Comité International des Sciences Historiques (L'œuménisme des historiens. Histoire des congrès internationaux d'Histoire et du Comité International des Sciences Historiques), Göttingen, Vandenhoeck \& Ruprecht, 1987, p. 87.

${ }^{33}$ TrOELTSCH, Ideen von 1914 (Idées de 1914), p. 616.
} 